\title{
Desulfurization of Industrial Water-Alkaline Solutions and Receiving New Plastic Oils
}

\author{
Olga Khudoyarova', Olga Gordienko², Alina Blazhko', \\ Tetiana Sydoruk², Anatoliy Ranskiy² \\ 1 Vinnytsia Mykhailo Kotsiubynskyi State Pedagogical University, Ostrozhskogo street 32, Vinnytsia, 21100, \\ Ukraine \\ 2 Vinnytsia National Technical University, Khmelnytske road 95, Vinnytsia, 21021, Ukraine \\ *Corresponding author's e-mail: helgakhudoyarova@gmail.com
}

\begin{abstract}
The research on the reagent extraction of sulfides from water-alkaline solutions was presented in the article with the purpose of their possible practical use as a component of special high-temperature grease in high-friction steam. The conditions for obtaining pentasulfide sodium and its adsorption on mixed sorbents $(\mathrm{AC}+\mathrm{K})$ were established. The complex-coordination mechanism of copper (II) sulfide formation and the method of obtaining new carbon-sulfur-containing plastic oils were offered, as well as their tribological properties in the friction pairs of roller four-row bearings were investigated.
\end{abstract}

Keywords: water-alkaline solutions, desulfurization, sorbent, greases.

\section{INTRODUCTION}

Environmental pollution by solid industrial waste and wastewater from the chemical, coke, refining, mining, and metallurgical industries puts a significant burden on the environment. This is due to the presence of toxic and highly toxic chemical compounds - sulfides, hydrosulfides, mercaptans and halogenated organic compounds, as well as heavy metals with low MPC values in their composition. Therefore, for example, the sulfur-containing wastewater of a number of chemical industrial industries are subject, as a rule, to neutralization with alkaline solutions, utilization of spent sulfide-alkaline solutions of crude oil purification from the sulfur-containing compounds by reagent treatment, according to the general scheme which was investigated in work [Ranskiy A. et al. 2012]:

$$
\begin{gathered}
\mathrm{NaHS}+\mathrm{NaOH} \rightarrow \mathrm{Na}_{2} \mathrm{~S}+\mathrm{H}_{2} \mathrm{O} \\
\mathrm{Na}_{2} \mathrm{~S}+(x-1) \mathrm{S} \rightarrow \mathrm{Na}_{2} \mathrm{~S}_{x} \\
\mathrm{Na}_{2} \mathrm{~S}_{x}+\mathrm{CuSO}_{4} \rightarrow \mathrm{CuS}_{x} \\
+\mathrm{Na}_{2} \mathrm{SO}_{4}, x=2-5
\end{gathered}
$$

The resulting copper (II) CuSx polysulfide can be used as a component of high temperature grease in high friction vapors.

Thus, the high-load friction units of modern machines and mechanisms are characterized by extended high-temperature operating ranges, increasing their contact and vibration loads. In addition, plastic oils are used in friction units operating in deep vacuum, in redox gas environments, in contact with water, under severe radiation and many other specific operating conditions.

A lot of operating conditions require the development and use of a large number of specialized plastics oils that differ in composition, function and operational characteristics.

Today, a large amount of applied research explains the connection of the experimental materials obtained with the structure of the structural framework of plastic oils, their rheological and operational characteristics, the physicochemical properties of the dispersion medium and the dispersed phases. In a number of works 
it was shown [Anisimova 2018; Mang 2010] that the performance characteristics of plastic oils can be significantly improved by the introduction of functional additives (anti-wear, antiantifriction), fillers and other additives into their composition.

On the basis of the above, the new carbonsulfur-containing plastic oils were obtained and their tribological properties in the friction pairs of roller four-row bearings were investigated (GOST 8419-75).

\section{MATERIALS AND METHODS}

A mixture of activated carbon and diatomaceous earth $(\mathrm{AC}+\mathrm{K})$ production of soft drinks (PF "Panda", Vinnytsia) was used as the sorbents, regenerated according to the methods given in works [Ranskiy et al. 2019a; Ranskiy et al. 2019b].

Desulfurization of industrial wastewater containing sulfide and hydrosulfide ions was performed by using model solutions of sodium sulfide and hydrosulfide at the concentrations close to the wastewater of the Kremenchug Refinery (PJSC "Ukrtatnafta", Ukraine).

The content of sulfur ions $\left(\mathrm{S}^{2-}, \mathrm{HS}^{-}\right)$in aqueous solutions, which were desulfurized, was determined by iodometric titration according to the procedure described in work [Ranskiy et al. $2019 \mathrm{c}]$. Sodium pentasulfide $\mathrm{Na}_{2} \mathrm{~S}_{5} \cdot 5 \mathrm{H}_{2} \mathrm{O}$ was obtained using the classical method, which is given below, and its purity was confirmed with the method of X-ray phase analysis (Fig. 1).

The model solutions containing sulfide and pentasulfide ions were purified by adsorption using mixed sorbents: activated carbon and diatomaceous earth $(\mathrm{AC}+\mathrm{K})$ in a laboratory unit that included a high-speed magnetic stirrer VELP AREC (VELP scientifica, colobrotone) Liebig's refrigerator.

The surface studies of sorbents $(\mathrm{AC}+\mathrm{K})$ were conducted by using a Dron-2 diffractometer in monochromatized Co-K $\alpha$ radiation $(\lambda=1.7902$ $\AA$ ). X-ray analysis / X-ray data were identified by comparing interplanar distances $(\mathrm{d}, \AA)$ and relative intensities (Iotn-I / $\mathrm{I}_{0}$ ) of the experimental curve with PCPDFWIN electronic records [JCPDS-PDF database 1999].

The tests of the new carbon-sulfur-containing plastics oils were carried out at Biolog Ltd. (Kherson, Ukraine) in the secondary polymer raw materials processing plant, namely on SM-PD
1500 660/660 LM1 rollers manufactured at the Yaroslavl Polymer Engineering Plant. The tests compared the loading (Pmax) and temperature characteristics of the friction unit under the recommended standard operating conditions of solidid (GOST 1033-79) with the actual operating conditions of the developed carbon-sulfur-containing plastic oils.

\section{Preparation of sodium pentasulfide $\mathrm{Na}_{2} \mathrm{~S}_{5} \cdot 5 \mathrm{H}_{2} \mathrm{O}$}

A $100.0 \mathrm{~g}$ of $12 \%$ sodium sulfide solution was placed in a flat bottom flask equipped with a thermometer and reflux condenser, and then $25 \mathrm{~g}$ of fine elemental sulfur and $6 \mathrm{~g}$ of sodium hydroxide were gradually added with stirring of the reaction mass with a magnetic stirrer. The reaction mass was kept under stirring and at a temperature of $120^{\circ} \mathrm{C}$ for $45-60 \mathrm{~min}$. The reaction mass was then cooled, the first precipitated sodium tetrasulfide crystals were filtered off and the resulting filtrate was cooled to $5{ }^{\circ} \mathrm{C}$ and left in the dark for several days. The precipitated red-orange crystals were filtered off and dried in a desiccator. The yield was $9.30 \mathrm{~g}$ (63\%wt.), Mp was $250-255^{\circ} \mathrm{C}$.

\section{Modification of the surface of the mixture of activated carbon and diatomaceous earth $(A C+K)$ with pentasulfide sodium}

To $10 \mathrm{~g}$ of a mixture of activated carbon sorbents and diatomaceous earth $(\mathrm{AC}+\mathrm{K})$, $100 \mathrm{ml}$ of a $5 \%$ aqueous-alcoholic solution of pentasulfide sodium was added. The resulting mixture was vigorously stirred on a magnetic stirrer $(\mathrm{n}=350 \mathrm{rpm})$ for $45 \mathrm{~min}$ at $20{ }^{\circ} \mathrm{C}$. The mixture was then filtered and the degree of adsorption/extraction of pentasulfide sodium from the original aqueous-alcoholic solution was determined. For the modified surface (MS1) obtained, the degree of extraction of pentasulfide sodium was $73 \%$.

\section{Modification of the previously obtained surface (MS-1) with copper (II) sulfide}

To $20 \mathrm{~g}$ of a modified mixture of sorbents $(\mathrm{AC}+\mathrm{K})$ sodium pentasulfide (MS-1 surface), $100 \mathrm{ml}$ of $28 \%$ copper (II) sulfate was added through a dropping funnel. The resulting mixture was vigorously stirred on a magnetic stirrer 
( $\mathrm{n}=350 \mathrm{rpm}$ ) for $45 \mathrm{~min}$ at $20^{\circ} \mathrm{C}$. The mixture with the modified surface (MS-2) was filtered, washed thoroughly with distilled water and dried in a desiccator, and then used as a component of new plastic oils (Table 1).

\section{Preparation of lubricating compositions of the PL series (general method)}

The lubricating compositions were prepared using a high-speed magnetic stirrer and heated to a constant mass of $70-90^{\circ} \mathrm{C}$. To a mixture of $42.7 \mathrm{~g}$ of Constalin grease 1-13 and industrial oil I-40, $15.0 \mathrm{~g}$ of boron-containing organic additive ("Boran") was added, and the mixture was stirred using a magnetic stirrer at $40-45{ }^{\circ} \mathrm{C}$ for $30 \mathrm{~min}$. Afterwards, $23.0 \mathrm{~g}$ of a mixture of sorbents $(\mathrm{AC}+\mathrm{K})$ having a modified surface (MS-2) was gradually added. Further rapid stirring was performed for 30-40 min, gradually raising the temperature of the reaction mass to $70-75^{\circ} \mathrm{C}$.
In the final step, $15.0 \mathrm{~g}$ of graphite and $4.3 \mathrm{~g}$ of oleic acid were added portionwise. The reaction mass was kept until complete homogenization, and then cooled. The obtained new carbon-sulfurcontaining grease (Table 1) was ready for the tribotechnical research.

\section{RESULTS}

In order to enable the practical use of sulfides, their reagent extraction from aqueous-alkaline solutions was investigated according to the general scheme (1). In the modified version, the reaction was carried out by using a turbulent mode of stirring the reaction mass, which allowed to obtain sodium pentasulfide $\mathrm{Na}_{2} \mathrm{~S}_{5} \cdot 5 \mathrm{H}_{2} \mathrm{O}$ with a yield of $63 \%$. In the X-ray diffraction pattern (Fig. 1), in addition to the peaks characteristic of the $\mathrm{Na}_{2} \mathrm{~S}_{5}$ parent compound, there are peaks belonging to a small amount of the $\mathrm{Na}_{2} \mathrm{~S}_{4}$ impurity.

Table 1. The composition of the investigated lubricating compositions of the PL series

\begin{tabular}{|c|l|c|c|c|c|c|}
\hline No. & lomponents, $\%$ & PL-1 & PL-2 & PL-3 & PL-4 & PL-5 \\
\hline 1 & Constalin oil 1-13 & 30.0 & 30.0 & 28.5 & 28.5 & 15.5 \\
\hline \multirow{3}{*}{2} & Industrial Oil: & & & & & \\
\cline { 2 - 6 } & I-20 & - & 15.0 & 14.2 & 14.2 & \\
\cline { 2 - 6 } & I-40 & & & & & 12.4 \\
\cline { 2 - 6 } & I-50 & 8.0 & - & - & & 15.5 \\
\hline 3 & I-20 + benzthiazole 0.1\% & 15.0 & 15.0 & 14.3 & 23.0 & 23.3 \\
\hline 4 & Cuprum (II) polysulfide & 15.0 & 20.0 & 14.2 & 15.0 & 2.3 \\
\hline 5 & Boron-containing organic additive & - & - & 5.1 & 4.3 & 31.0 \\
\hline 6 & Oleic acid & 25.0 & 20.0 & 23.7 & 15.0 & 100 \\
\hline 7 & Graphite & 100 & 100 & 100 & 100 & \\
\hline 8 & Total & & & & & \\
\hline
\end{tabular}

Note: PL-1 - PL-4 lubricating compositions have undergone industrial testing at Biolog LLC (Kherson, Ukraine), and PL-5 lubricating composition at DneproPress Heavy Press Plant (Dnipro, Ukraine).

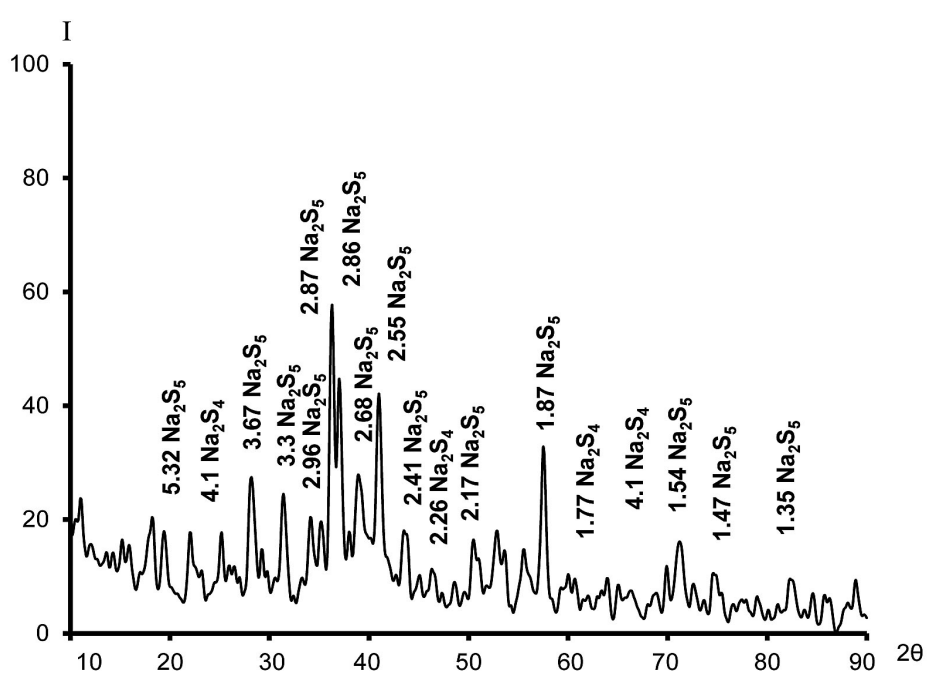

Fig. 1. X-ray diffraction pattern of $\mathrm{Na}_{2} \mathrm{~S}_{5} \cdot 5 \mathrm{H}_{2} \mathrm{O}$ compound 


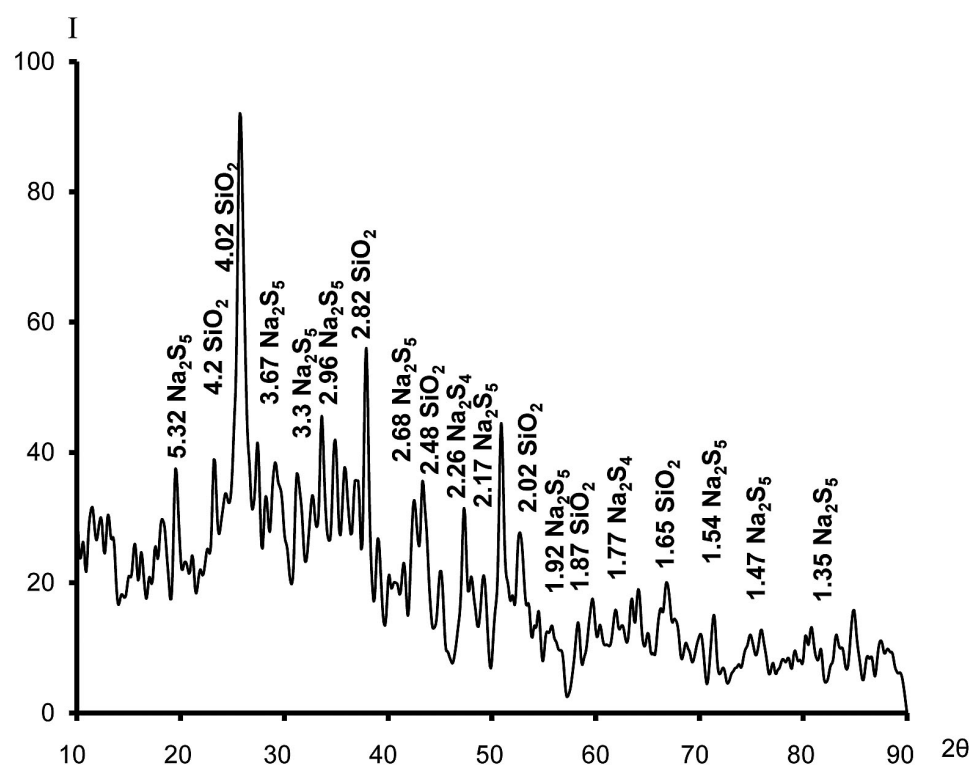

Fig. 2. X-ray diffraction pattern of $\mathrm{Na}_{2} \mathrm{~S}_{5} \cdot 5 \mathrm{H}_{2} \mathrm{O}$ compound on modified MS-1 surface

In the course of processing the reaction mass, the main product was separated from the cyclotetrasulfan by step crystallization.

It should be noted that the conduct of laboratory experiments is accompanied by the hydrolysis of sodium sulfides with the formation of toxic hydrogen sulfide. The latter was withdrawn from the reaction zone with inert gas $\left(\mathrm{N}_{2}\right)$ followed by bubbling through a $10 \% \mathrm{NaOH}$ solution.

Subsequently, the aqueous-alcoholic solution of pentasulfide sodium was subjected to sorption by mixed sorbents $(\mathrm{AC}+\mathrm{K})$ in order to not only purify the aqueous solution from sodium polysulfides, but also to create a matrix for their topochemical reaction with copper salts (II). However, it was unable to obtain $\left[\mathrm{Cu}\left(\mu-\mathrm{S}_{5}\right)\right]_{2}$ or $\mathrm{CuS}_{2}$ (an analogue of the main industrial component of $\mathrm{MoS}_{2}$ plastic oils). Instead, the formation of copper (II) sulfide and, most of all, cyclotetrasulfane $\mathrm{S}_{4}$ was established. The X-ray diffraction pattern (Fig. 2) shows the data confirming the adsorption of sodium polysulfides from alkaline solutions by mixed sorbents $(\mathrm{AC}+\mathrm{K})$.

The practical use of the obtained polysulfides of metals: $\mathrm{Na}_{2} \mathrm{~S}_{5}, \mathrm{Na}_{2} \mathrm{~S}_{4},\left[\mathrm{Cu}\left(\mu-\mathrm{S}_{5}\right)\right]_{2}$, as constituents of new carbon-sulfur-containing oils, was determined by their degree of sulfidity [Sangalov et al. 2013], which governs their main physicochemical, thermal and thermodynamic properties. Thus, it was shown that sodium pentasulfide is not stable and not expected by our inter-chain polysulfide exchange, conducting a topochemical reaction on a solid surface $(\mathrm{AC}+$ $\mathrm{K}$ ), and the destruction of the polysulfide chain with the formation of copper (II) sulfide and cyclic sulfur according to the scheme (2).

Figure 3 shows the X-ray diffraction pattern of the topochemical reaction of copper (II) sulfide and cyclotetrasulfane formation, on which, the presence of a small amount of copper (II) oxide and elemental sulfur corresponding to the passage of the topochemical reaction according to the scheme (2) was observed besides the specified copper (II) sulfide.

In the study of the formation of modified surfaces of MS-1 and MS-2 on sorbents (AC $+\mathrm{K}$ ) using, respectively, $\mathrm{Na}_{2} \mathrm{~S} \cdot 5 \mathrm{H}_{2} \mathrm{O}$ and $\mathrm{CuSO}_{4} \cdot 5 \mathrm{H}_{2} \mathrm{O}$ determining the rate of adsorption/diffusion of the investigated substances at their active centers. In the case of modified surface MS-1, the latter has a large number of sulfur atoms on its surface, which act as sulfide ligands of the $\mathrm{Cu}^{2+}$ ions and ensure their rapid adsorption by a complex coordination mechanism:

It is obvious that the formation of the abovementioned plane complex takes place only in the

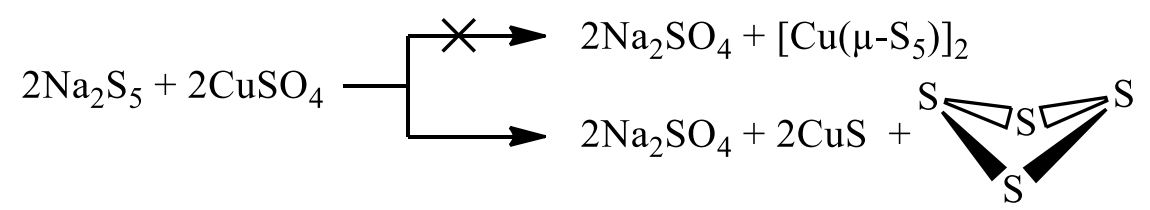




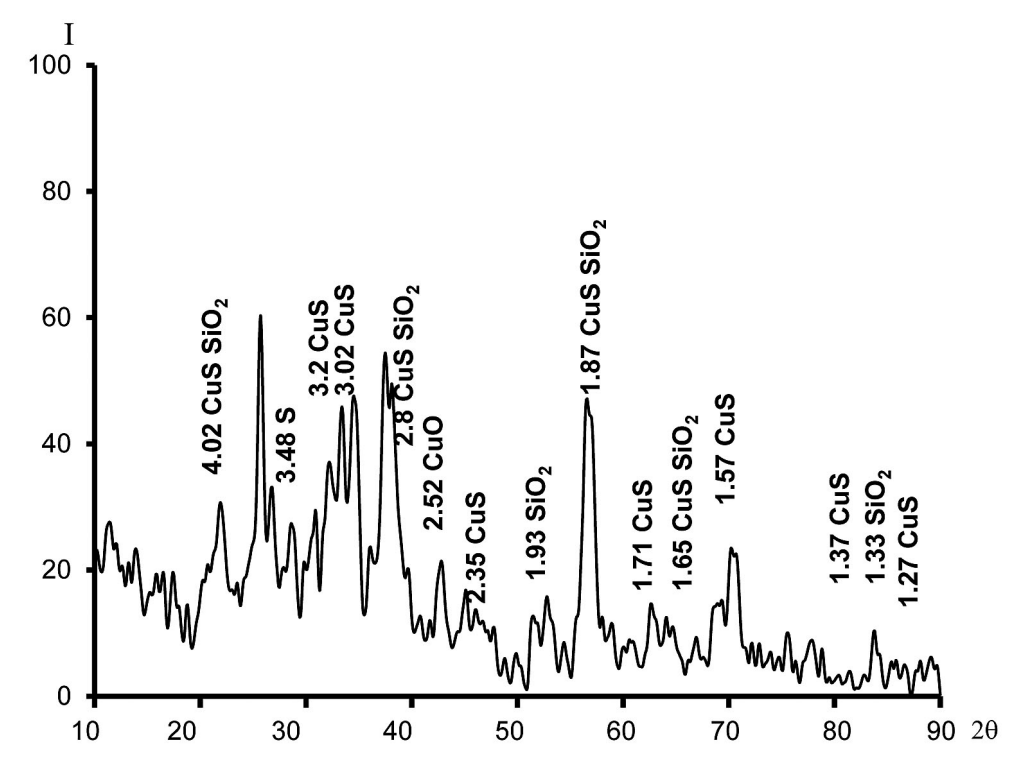

Fig. 3. X-ray diffraction pattern of CuS compound on the modified surface of MS-2

first stage, and then its destruction occurs with the formation of copper (II) sulfide and elemental sulfur, which is confirmed by the results of X-ray phase investigations (Fig. 3).

It should be noted that a similar mechanism pertaining to the adsorption of mercury (II) ions by the sulfur-containing sorbents was investigated in [Redinova et al. 2013].

The operational studies of friction pairs showed that the use of, for example, solidol is only possible at temperatures not exceeding $50-60^{\circ} \mathrm{C}$, whereas the actual temperature in the friction pairs reaches $85-90{ }^{\circ} \mathrm{C}$. Under these conditions, the solidol lubricant was leaking out, and in the friction pair, there was a risk of the four-row roller bearings being jammed and decommissioned. When using the plastic oils we have developed (see table), the temperature at the friction units did not exceed the standard values in accordance with GOST 1033-79, and the surface of the roller bearings remained clean, smooth, free of rolls and cracks after 12 months of preventive observations.

It should be noted that the investigated friction pairs are characterized by high contact loads. Therefore, in the friction zone of copper (II) sulfide, elemental sulfur and high contact loads, formation and cuprum (II) of polysulfide are possible: $\mathrm{CuS}+\mathrm{nS} \rightarrow \mathrm{CuS}_{\mathrm{n}+1}, \mathrm{n}=1-4$.

Thus, it can be stated that the developed new carbon-sulfur-containing plastic oils have high thermal and anti-wear properties and expand the range of special plastic oils.

\section{CONCLUSIONS}

1. The adsorption of mixed sorbents $(\mathrm{AC}+\mathrm{K})$ of aqueous-alcoholic solution of pentasulfide sodium was investigated and the degree of extraction of the latter was found to be $73 \%$. Modified sodium pentasulfide, the solid surface of the formed sorbents (MS-1), which was formed, was used as a component in the topochemical reaction to obtain the sulfur-containing compounds.

2. The topochemical reaction of formation on the surface of mixed sorbents (AC $+\mathrm{K}$ ) copper (II) sulfide was investigated. A complex-coordination mechanism for the formation of copper (II) sulfide was proposed, which was confirmed by the data of the X-ray phase studies of the modified MS-2 surface.

3. New carbon-sulfur-containing plastic oils, consisting of the obtained modified copper (II) sulfide-mixed sorbents $(\mathrm{AC}+\mathrm{K})$, were investigated. High tribotechnical properties of the investigated plastic oils of the PL series were established to ensure stable operation of hightemperature and high-load friction units.

\section{REFERENCES}

1. Anisimova A.S. 2018. Study of the effect of additives on the properties of sulfonate plastic lubricants: dis. ... cand. tech. sciences: 05.17.07 "Chemical technology of fuel and high-energy substances". Moscow, 170. 
2. JCPDS-PDF database - International Center for Diffraction Data.PCPDFWIN. 1999, 2.02.

3. Mang T. 2010. Lubricants. Production, application, properties: Reference book, per. from. English Edited by V.M. Shkolnikov. SPb . CEP Profession, 944.

4. Ranskiy A., Evseyeva M., Gordienko O., Titov T., Polonets O. 2012. Utilization of alkaline-sulfide solutions in industrial production. Bulletin of VPI, $1,30-33$.

5. Ranskiy A., Khudoyarova O., Gordienko O., Kryklivyi R., Titov T. 2019a. Utility Model Patent 134391 (51) IPC C01B 32/30, C01B 32/36, B01J $20 / 34$. The method of regeneration of a mixture of activated carbon and kieselguhr from organic pollutants, 9.

6. Ranskiy A., Khudoyarova O., Gordienko O., Titov T., Kryklivyi R. 2019b. Regeneration of Sorbents
Mixture After the Purification of Recycled Water in Production of Soft Drinks. Journal of Water Chemistry and Technology, 5(41), 318-321.

7. Ranskiy A., Khudoyarova O., Gordienko O., Titov T.,Tserklevych D., Korinenko B. 2019c. Utility model patent 139177 (51) of IPC C01B 17/22, C01B $17 / 16$, B01J 20/34. The method of purification of industrial wastewater from sulfide and hydrosulfide ions, 24.

8. Redinova A.V., Grabel'nykh V.A., Levanova E.P., Korchevin E.P. 2013. Extraction of heavy metal ions agueons solutions of sulfurcontaining polymeric sorbents. Vestnik Ir STU, 1, 113-116.

9. Sangalov Y., Karchevskyi S., Ionov V. 2013. Polysulfides are the most important representatives of high-sulfur compounds. Bulletin of the Academy of Sciences of the Republic of Belarus, 1(18), 15-24. 